\section{ORIGINAL RESEARCH}

W.S. Tae

S.S. Kim

K.U. Lee

E.C. Nam

J.W. Choi

J.I. Park

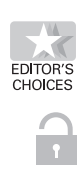

\title{
Hippocampal Shape Deformation in Female Patients with Unremitting Major Depressive Disorder
}

BACKGROUND AND PURPOSE: The hippocampal atrophy of MDD has been known, but the region shape contractions of the hippocampus in MDD were inconsistent. Spheric harmonic shape analysis was applied to the hippocampus in female patients with unremitting MDD to evaluate morphometric changes of the hippocampus.

MATERIALS AND METHODS: Shape analysis was performed by using T1-weighted MR imaging in 21 female patients with MDD and 21 age- and sex-matched healthy controls. Manually segmented hippocampi were parameterized, and the point-to-point-based group difference was compared by using the Hotelling T-squared test. The partial correlation analyses were tested between clinical variables and shape changes.

RESULTS: Both hippocampal volumes were small in patients with MDD compared with healthy controls, and the right hippocampal volume was negatively correlated with the number of episodes at marginal significance. Regional shape contractions were found in the ambient gyrus, basal hippocampal head, posterior subiculum, and dorsal hippocampus of the left hemisphere. The right hippocampus showed a similar pattern but was less atrophic compared with the left hippocampus. A negative correlation was found between the HDRS and shape deformation in the CA3, ambient gyrus, posterior subiculum, and gyrus fasciolaris of the left hippocampus.

CONCLUSIONS: We showed atrophy and regional shape contractions in the hippocampi of patients with MDD, which were more dominant on the left side. The causes of hippocampal damage could be the hypersecretion of glucocorticoids contributing to neuronal death or the failing of adult neurogenesis in the dentate gyrus.

\begin{abstract}
ABBREVIATIONS: $A G=$ ambient gyrus; $A N C O V A=$ analysis of covariance; Apoe $=$ apolipoprotein $E_{;}$Avr. Surf. = average statistic across the surface; $\mathrm{BDI}=$ Beck Depression Inventory; $\mathrm{CA}=$ cornu ammonis; FDR = false discovery rate; HDRS = Hamilton Depression Rating Scale; ICC = intraclass correlation coefficient; ICV = intracranial cavity volume; $\mathrm{LH}=$ left hippocampus; MDD = major depressive disorder; $\mathrm{N \#} \mathrm{=} \mathrm{the} \mathrm{subject} \mathrm{number} \mathrm{of} \mathrm{each} \mathrm{healthy} \mathrm{control} \mathrm{subject;} \mathrm{P \#} \mathrm{=} \mathrm{the} \mathrm{subject}$ number of each patient with MDD; PDM = point distribution model; $\mathrm{RH}=$ right hippocampus; SCID = Structured Clinical Interview for DSM-IV; SPHARM = spheric harmonic basis functions; Sub = subiculum; TFE $=$ turbo field echo; $\mathrm{VOI}=$ volume of interest
\end{abstract}

$\mathbf{T}_{\mathrm{i}}^{\mathrm{h}}$ he hippocampus is a part of the limbic system and plays an important role in short-term memory, the formation of new memories, the retrieval of older memories, spatial navigation, learning, contextual-fear conditioning, and neuroendocrine regulation. ${ }^{1,2}$ The hippocampus is a specific region producing new neurons throughout adult life, and hippocampal formation has been considered an important causal factor for episodes of clinical depression. ${ }^{3}$ The hypersecretion of glucocorticoids, the adrenal steroids secreted during stress, contributes to the hippocam-

Received August 3, 2010; accepted after revision September 10.

From the Neuroscience Research Institute (W.S.T., S.S.K., K.U.L., E.C.N., J.W.C., J.I.P.), Kangwon National University School of Medicine, Chuncheon, Korea; and Medical Science and Engineering Research Center for Bioreaction to Reactive Oxygen Species (S.S.K.), Seoul, Korea.

This work was supported by the Korea Science and Engineering Foundation grant funded by the Korean Ministry of Education, Science and Technology (No. 20090091346) and by the Korea Research Foundation Grant funded by the Korean Government (KRF-2008-359-E00009).

Please address correspondence to Sam Soo Kim, MD, Department of Radiology, Kangwon National University Hospital, 17-1 Hyoja 3-dong, Chuncheon, Kangwon-do 200-947, Korea; e-mail: samskim@kangwon.ac.kr

Indicates open access to non-subscribers at www.ajnr.org

DOI 10.3174/ajnr.A2367 pal atrophy found in certain subtypes of depression. ${ }^{4}$ Hippocampal volume reductions in patients with depression have been summarized in meta-analyses, ${ }^{5,6}$ which have concluded that patients with depression and unremitting depression of long duration, the presence of abuse, and unipolar depression show a greater reduction in hippocampal volume.

Shape analysis is a recently developed method for the statistical evaluation of morphometric changes of brain subregions. The volumetry of the hippocampus only provides the measured volume information as a mass, and though the hippocampal volumetry could be advantageous in the detection of mild hippocampal volume changes throughout the whole hippocampus, it might also be valuable to screen the atrophic or hypertrophic subregions of the hippocampus. Shape analysis allows statistical assessment of the subregional anatomy of morphologically changed areas with 3D modeling of the hippocampus so that contracted or expanded subregions of the hippocampus can be identified. ${ }^{7,8}$ The hippocampus consists of various subregions such as CA1 through CA4, the dentate gyrus, fimbria, ambient gyrus, and subiculum; these subregions are interconnected, and each has a different structural or functional connection with the other brain subregions. Shape deformations of hippocampal subregions provide important 


\begin{tabular}{|c|c|c|c|c|c|}
\hline \multicolumn{6}{|c|}{ Clinical variables and the results of MR imaging volumetry } \\
\hline & $\begin{array}{l}\text { Healthy Controls } \\
\text { (SD) }\end{array}$ & $\begin{array}{l}\text { MDD Patients } \\
\text { (SD) }\end{array}$ & $T$-Test, $P$ & ANCOVA, $P^{\mathrm{a}}$ & Avr. Surf., $P$ \\
\hline No. of patients & 21 & 21 & - & - & - \\
\hline Age (yr) & $42.3(10.20)$ & $41.7(11.0)$ & .84 & - & - \\
\hline Onset age of depressive episode (yr) & - & $33.2(3.0)$ & - & - & - \\
\hline Number of depressive episodes (yr) & - & $3.9(3.3)$ & - & - & - \\
\hline IIIness duration (yr) & - & $6.7(5.6)$ & - & - & - \\
\hline HDRS & - & $16.8(7.70)$ & - & - & - \\
\hline $\operatorname{ICV}\left(\mathrm{cm}^{3}\right)$ & $1395.9(120.48)$ & $1393.6(170.84)$ & .959 & - & - \\
\hline \multicolumn{6}{|l|}{ Hippocampal volumes $\left(\mathrm{cm}^{3}\right)$} \\
\hline Right & $3.24(0.277)$ & $2.97(0.392)$ & .013 & .003 & .080 \\
\hline Left & $3.18(0.295)$ & $2.79(0.441)$ & .002 & .001 & .00075 \\
\hline Asymmetry of hippocampal volume ${ }^{\mathrm{b}}$ & 0.212 & 0.0001 & - & - & - \\
\hline
\end{tabular}

a ANCOVA covariate with ICV and age.

b Paired $t$ test between the right and left hippocampal volumes in each group.

information for understanding the hippocampal abnormalities of patients with neuropsychiatric diseases.

The purpose of this study was to determine regional shape deformation in the hippocampi of patients with unremitting MDD and to investigate the relationship between local shape deformation and clinical variables by means of shape analysis.

\section{Materials and Methods}

\section{Subjects}

The subjects enrolled in this study had also been included in the previous hippocampus validation study, described in detail elsewhere. ${ }^{9}$ Twenty-one female patients with unremitting MDD (18-60 years of age) were recruited from the patients of the Department of Psychiatry at our hospital. Additionally, 21 healthy female subjects (24-58 years of age), matched with the patients with MDD for age, handedness, and parental socioeconomic status, were recruited from the community (Table). The study was approved by the institutional review board of the hospital.

Patients were only included if they fulfilled the criteria for MDD based on the SCID of the Diagnostic and Statistical Manual of Mental Disorders, Fourth Edition (American Psychiatric Association). ${ }^{10}$ The severity of depressive symptoms was assessed by using the $\mathrm{BDI}^{11}$ and the 17-item HDRS. ${ }^{12}$ Both the SCID and HDRS were administered by an experienced psychiatrist. Patients were excluded from the study if they had a history of childhood trauma or other major Axis I disorders, including bipolar disorder, schizophrenia, schizoaffective disorder, claustrophobia, or a current or past history of alcohol or substance abuse or dependence. All patients were taking antidepressant medication, but none were taking antipsychotics or mood stabilizers. Control subjects had no personal or family history of Axis I disorders. The mean age of the first depressive episode, the mean number of lifetime depressive episodes, and mean illness duration are detailed in the Table.

\section{MR Imaging Acquisition}

All of the MR images were obtained with a 1.5T scanner (Gyroscan ACS-NT; Philips Healthcare, Best, the Netherlands). Coronal 3D T1weighted TFE MR images were obtained with the following scanning variables: section thickness $=1.3 \mathrm{~mm}, 160$ sections, scanning time $=$ 10 minutes 13 seconds, TR/TE $=10 / 4.3 \mathrm{~ms}$, number of signal intensity averages $=1$, matrix $=256 \times 256$, FOV $=22 \times 22 \mathrm{~cm}, 160$ sections, and $8^{\circ}$ flip angle. Coronal sections were obtained perpendicular to the long axis of the anterior commissure to the posterior commissure in the midsagittal plane. The final voxel size was $0.86 \times$ $0.86 \times 1.30 \mathrm{~mm}(x \times y \times z)$.

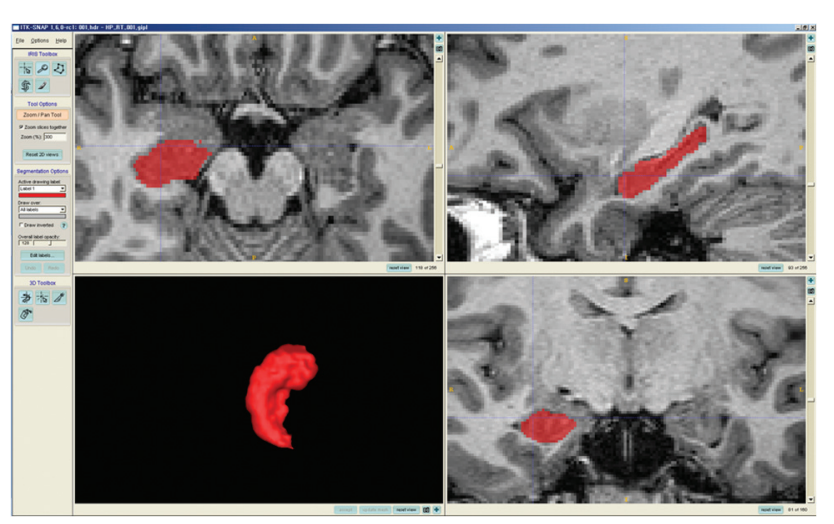

Fig 1. Hippocampus labeling. With ITK-SNAP software, the whole hippocampus is labeled from the anteriormost head to the posterior tail, and the labeled hippocampus is segmented and saved as binary images for the shape analysis.

\section{Intracranial Volume Measurement}

The ICVs of all subjects were measured manually; the reproducibility of ICV has been previously reported (Cronbach $\alpha=0.996){ }^{9}$ ICVs were used to correct for variations in individual brain size as a basis for shape analysis and hippocampal volumetry.

To measure the ICV, we reconstructed the original T1-weighted TFE MR imaging data to create 5-mm-thick sagittal images. The cerebrum, cerebellum, and midbrain were included in the ICV volume. The lateral limits of the ICV were defined as the right- and leftmost sections of the brain parenchyma on sagittal images, and the lower tip of the cerebellum was defined as the lower limit. Using the established measurement criteria, we traced the dura mater of the cerebrum, the cerebellum, and the medulla manually. ${ }^{13}$

\section{Hippocampus Boundary Determination}

The boundary of the hippocampus was determined by using ITK-SNAP 1.6 (www.itksnap.org). ${ }^{14}$ This software provides an interactive labeling function with simultaneous coronal, sagittal, and axial views and concurrently provides surface rendering of the VOI. The boundary of the hippocampus could be traced in any view, the VOI of the hippocampus could be modified easily, and the boundary between the amygdala and the hippocampal head could be separated accurately (Fig 1).

Coronal T1 MR imaging data were loaded into ITK-SNAP and magnified by $300 \%$. Using the polygon and paintbrush tools, we traced the hippocampus manually from the anteriormost head to the posterior tail, including the $\mathrm{CA}$, gyrus dentatus, hippocampus, subiculum, ambient gyrus, and the gyrus fasciolaris. The fasciola cinerea was also included in the shape analysis. The anteriormost boundary of the 


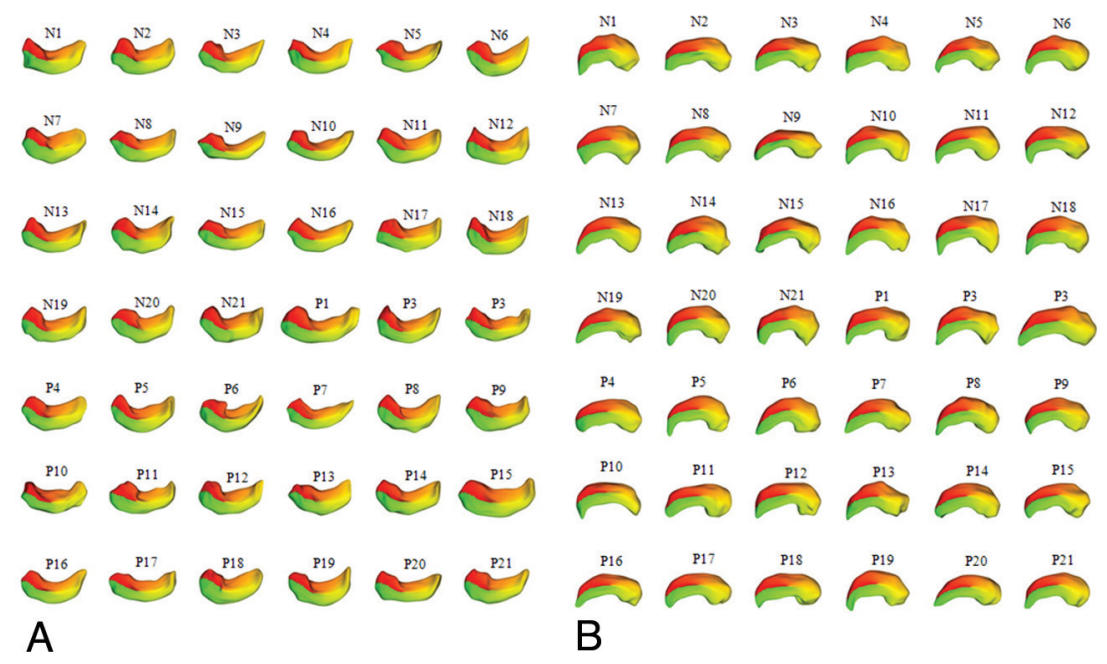

Fig 2. SPHARM-PDM images of the hippocampus. A, Left hippocampus. B, Right hippocampus. The 84 binary images of all subjects' hippocampi were successfully converted to SPHARM-PDM images.

hippocampus was identified as the alveus, and suspected boundaries were identified in the coronal view, while the sagittal view displayed the pixel in the same position. The lateral border of the hippocampus was delineated against the entorhinal cortex by the upper margin of the white matter of the subiculum. The posterior end of the hippocampus was taken as the point at which the tail of the hippocampus disappeared. Sectors CA1 through CA4 of hippocampus proper, the dentate gyrus, ambient gyrus, subiculum, gyrus fasciolaris, and fasciola cinerea were included in the in-plane boundaries; the white matter, including the alveus and fimbria, was also part of the hippocampal definition. Hippocampus labeling was performed by 1 of the authors (W.S.T.) who is experienced in hippocampal anatomy and volumetry.

All MR imaging data from both healthy subjects and patients with MDD were mixed in the same directory before measurement, without clinical information. To test the reproducibility of hippocampus volumetry, we again measured both hippocampi of 5 healthy subjects and 5 patients with MDD. The ICCs (Cronbach $\alpha$ ) for hippocampal volumetry were 0.983 and 0.946 for the right and left hippocampus, respectively. ${ }^{15}$

\section{Hippocampal Shape Analysis}

The hippocampal shape analysis was processed by using the SPHARM-PDM and was based on a uniform-icosahedron subdivision of the spheric parameterization; the PDMs of all hippocampi were obtained. ${ }^{7}$ This boundary shape-analysis method can be used to express shape deformation. ${ }^{8,16}$

The segmented binary images of the left and right hippocampi were cropped to reduce the image size and were saved into separate files. The cropped images were resampled from a voxel size of $0.86 \times$ $0.86 \times 1.30 \mathrm{~mm}$ to $0.5 \times 0.5 \times 0.5 \mathrm{~mm}$. For the SPHARM computation, the parameters of subdivision and cutoff degree were set to 10 and 12, respectively. The correspondences across all surfaces were computed by aligning the first-order ellipsoids of all the parameterizations. The SPHARM-PDMs of all hippocampi (right, 42; left, 42) were successfully computed (Fig 2).

Before the shape analysis, the averaged left and right hippocampal templates were created separately by using all subjects' hippocampal images, and averaged templates were computed by averaging the $3 \mathrm{D}$ coordinates of corresponding surface points across the groups. ${ }^{16}$ All the triangulated meshes of both right and left hippocampi were spatially aligned to each template by using a rigid transform. Technical details of SPHARM-PDM shape analysis have been described in articles of previous developers. ${ }^{7,8,16}$

\section{Statistical Analysis}

Statistical analysis was performed with the Statistical Package for the Social Sciences, Version 11.5 (SPSS, Chicago, Illinois). Volume differences in ICV and both hippocampi between healthy subjects and patients with chronic MDD were examined by using a 2-tailed $t$ test and ANCOVA with the confounder of age; ICV was also obtained to correct for the effects of age or ICV. The asymmetry between right and left hippocampi was tested by a paired $t$ test. Partial correlations, controlling for age and ICV, were tested between each hippocampus and the following: 1) onset age of depressive episode, 2) illness duration, 3) number of depressive episodes, and 4) HDRS score. All tests were 2-tailed, and the level of significance was $P<.05$.

For the statistical method of shape analysis, the PDM consisted of coordinates corresponding to 1002 points (scaled distances from the template). With the scaled distances of the 1002 corresponding points, we tested differences between the 2 groups of surfaces by using the Hotelling T-squared test with an ICV correction. Using the results of this test, we created significance maps of uncorrected and FDR-corrected $P$ values corresponding to all 1002 points. For the global shape differences, the global average deviation between the mean surfaces of the 2 groups was tested with a 2-tailed $t$ test. With the Statistical Package for the Social Sciences, partial correlation analyses were performed between the scaled distances of the 1002 points and the clinical variables of each hippocampus: 1) onset age of depressive episode, 2) illness duration, 3) number of depressive episodes, and 4) HDRS, with the confounders of age and ICV. The significance maps of the right and left hippocampal analyses were displayed on the hippocampal template images of each side. The results were displayed at a significance level of $P<.05$ (uncorrected), and the clusters, including the voxels with a local maximum of $P<.005$ (uncorrected), were regarded as significant results. The color scale bar represented $P$ values.

\section{Results}

\section{Clinical Data and Volumetry}

The age and ICV distributions between the healthy female subjects and female patients with MDD were similar. Both 
absolute hippocampal volumes of the patients with MDD were smaller than those of the healthy controls. After correction for the effects of age and ICV, both hippocampal atrophies of patients with MDD were more significant (Table).

The left hippocampus was more atrophic than the right hippocampus (atrophy index $=2 \times$ [healthy $-\mathrm{MDD}$ ] / [healthy $+\mathrm{MDD}] \times 100$; right hippocampus, $8.7 \%$; left hippocampus, $13.1 \%$ ), and the hippocampal volumes of female patients with MDD were asymmetric (right $>$ left; $P=.0001$, 2-tailed paired $t$ test), whereas the hippocampal volumes of female healthy controls were not asymmetric $(P=.212$, 2-tailed paired $t$ test).

Neither left nor right hippocampal volumes in patients with MDD were correlated with age at depressive episode, illness duration, or HDRS (all, $P>.1$, two-tailed partial correlation, controlling for age and ICV). However, the right hippocampal volume showed a marginally significant $(P=.087$, 2 -tailed) negative correlation with the number of episodes, whereas the left hippocampal volume was not correlated with the number of episodes ( $P=.134$, two-tailed).

\section{Shape Analysis}

A globally contracted shape difference was observed in the left hippocampus of patients with $\operatorname{MDD}(P=.00075)$, and the right hippocampus of patients with MDD showed marginal global shape contraction $(P=.080)$.

In the left hippocampus, the results of shape analysis showed regionally contracted areas in the tip of the hippocampal head $(P=.003)$, ambient gyrus (rostral parahippocampal gyrus) $(P=.004)$, basal hippocampal head $(\mathrm{CA} 1)(P<.0001)$, subiculum of the hippocampal tail $(P=.002)$, and the 2 dorsal areas of the hippocampal head and tail, which correspond to CA2 and CA3 $(P<.0001)($ Fig $3 A)$.

In the right hippocampus of patients with MDD, the regions of the ambient gyrus $(P=.002)$, the lateral hippocampal head $(P=.004)$, and the subiculum of the hippocampal body $(P=.0008)$ were contracted compared with the hippocampus of healthy controls (Fig $3 A$ ).

No expanded areas were found in either hippocampus of patients with MDD compared with the hippocampi of healthy controls at the level of $P<.05$ (uncorrected).

After the correction for the multiple comparisons (FDRcorrected, $P<.05$ ), only the left hippocampus showed significant shape contractions in the dorsal areas of the hippocampal head and tail (CA 2-3) (FDR, $P=.006$ ), basal hippocampal head (CA1) (FDR, $P=.009$ ), and the subiculum of hippocampal tail (FDR, $P=.049$ ) (Fig 3B).

In the correlation analysis among the MDDs, HDRS scores, and the scaled distances of the 1002 points of the hippocampal mean PDMs, negatively correlated areas were observed in CA3 $(P=.001)$ of the hippocampal head, the basal hippocampal head $(P=.001)$, and the subiculum of the hippocampal tail $(P=.002)$. In the right hippocampus, the scaled distances in the areas of the subiculum of the hippocampal body $(P=.005)$ and the end of the hippocampal tail $(P=.004)$ were negatively correlated with the HDRS scores (Fig 4). However, the healthy controls did not show this correlation. Other clinical variables such as the onset age of the depressive episode or illness duration did not correlate with the hippocampal shape changes in patients with MDD. Because the results of hippocampal volume-
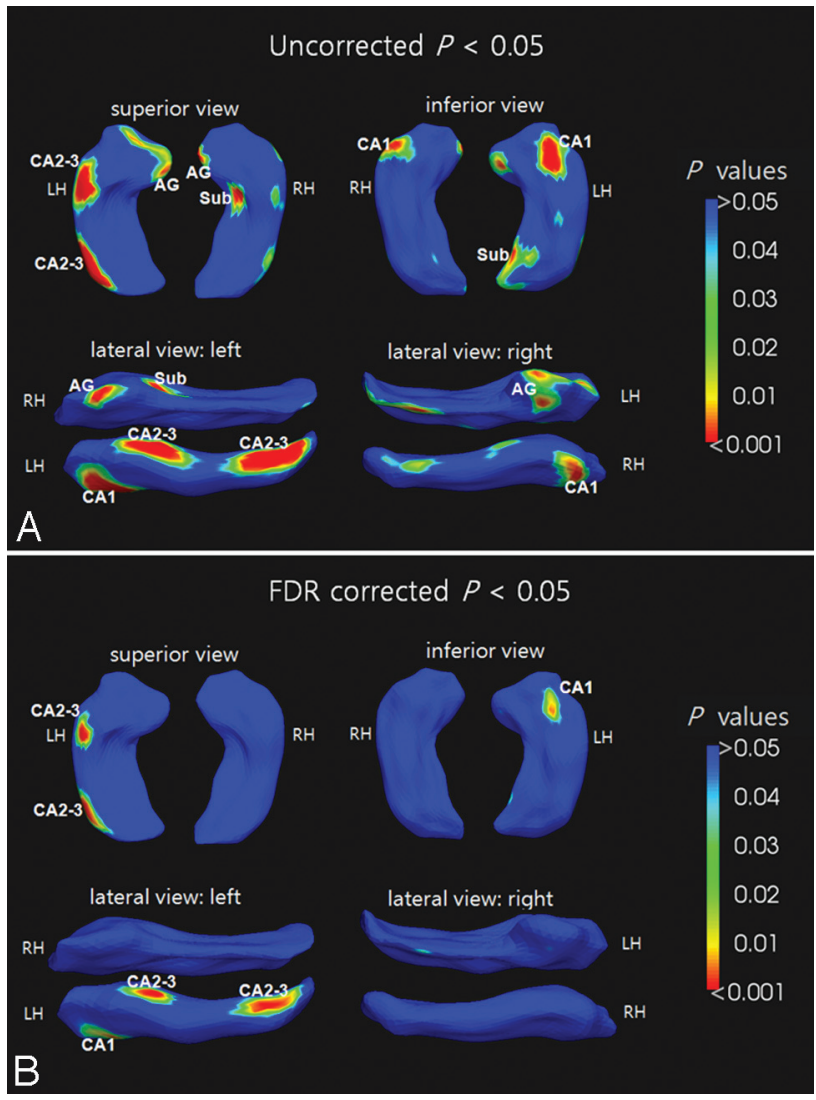

Fig 3. The results of shape analysis: female patients with chronic MDD versus healthy female controls. The significance maps display both averaged hippocampi at the uncorrected significance level of $P<.05(A)$ and corrected FDR of $P<.05(B)$. The color bar represents $P$ values.

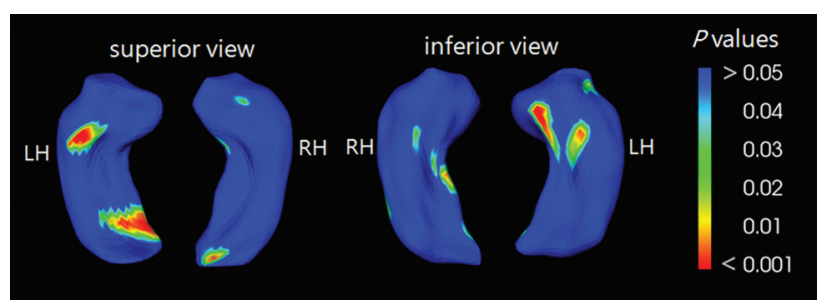

Fig 4. Negative correlations between the surface distance of the hippocampus and the HDRS. The significance maps are displayed for both averaged hippocampi at the uncorrected significance level of $P<.05$. The color bar represents $P$ values.

try showed significant volume reduction bilaterally, the results of the shape analysis with an uncorrected statistical level of $P<.05$ could provide intuitive information about more affected areas throughout the hippocampi of patients with MDD. ${ }^{17}$

\section{Discussion}

\section{ICV}

The ICV did not differ between healthy controls and patients with MDD, and this finding supports the hypothesis that ICV remains constant across the age spans. ${ }^{18}$ The estimation of the naïve brain volume before damage by diseases is a key process in structural MR imaging studies into the neuropsychiatry of diseases. ${ }^{19}$ Because the skull grows along the suture lines and the maximum skull cavity is determined by brain expansion during growth, ICV measurements indirectly provide a max- 
imum premorbid brain size and should be unaffected by atrophy due to neurodegeneration or aging. ${ }^{20}$ However, the use of total cerebral volume as the reference volume for the premorbid brain size is inappropriate, because the brain substructure and also total cerebral volume or total gray matter volume could be affected by neuropsychiatric disease and aging. ${ }^{18}$ The use of an incorrect ICV or the total cerebral volume of an atrophic brain as the reference volume could reduce the sensitivity of hippocampal volumetry and shape analysis.

\section{Hippocampal Volumetry}

In this study, we showed bilateral hippocampal volume reduction in patients with MDD, with left-dominant hippocampal atrophy in the absolute and in the age- and ICV-corrected hippocampal volumes. A rightward asymmetry of hippocampal volume in MDD, but not in healthy controls, was also observed. In addition, the possibility of a negative correlation between the number of depressive episodes and right hippocampal volume was suggested.

In our analysis, bilateral hippocampal volume reductions were found in patients with MDD. Many researchers have reported hippocampal atrophy in patients with $\mathrm{MDD},{ }^{5,6}$ and this hippocampal volume reduction has been found mainly in patients with multiple past episodes of depression, but it has not been observed in patients experiencing their first episode of depression. ${ }^{21}$ Reduced posterior hippocampal volume ${ }^{22}$ and progressive volume reduction in the right hippocampus in elderly depressed patients ${ }^{23}$ have also been reported. One recent meta-analysis concluded that hippocampal volume was lower in depressed patients than in healthy controls when the hippocampal volume was measured as a structure discrete from the amygdala and that illness duration seemed to be the critical factor in hippocampal atrophy. ${ }^{5}$ Another meta-analysis concluded that hippocampal volume was reduced in patients with unipolar depression, but patients with bipolar depression did not show a hippocampal volume reduction. ${ }^{6}$ In addition, the hippocampal atrophy of MDD was more obvious after controlling for ICV, and the atrophy was found to last for decades after the depression had remitted. ${ }^{1}$ Our results were in accord with these studies.

We also observed a rightward asymmetry of hippocampal volume in female patients with MDD, but not in healthy female controls, and this finding implies left-dominant hippocampal atrophy in patients with MDD. This asymmetric characteristic has been reported previously in the normal male brain, but not in the female brain. ${ }^{24,25}$ The hippocampal asymmetry of the healthy human is debatable, because some articles have reported hippocampal asymmetry, ${ }^{26,27}$ whereas others have reported hippocampal symmetry. ${ }^{28-31}$ The present study seems to support the hypothesis that hippocampal volume is not asymmetric in healthy women.

Except for the number of episodes of depression, none of the clinical variables examined were correlated with hippocampal volume. The number of episodes of depression showed a marginally significant negative correlation $(P=$ .087) with right hippocampal volume. Because the atrophy of hippocampal volume of patients with MDD is well-understood, ${ }^{5,6}$ the significance level could be increased with the use of 1-sided statistics $(P=.044)$, and this finding would then support the previously reported relationship between hippocampal volume and the number of episodes of depression. ${ }^{6}$

\section{Shape Analysis}

Although much research has reported hippocampal volume changes in patients with MDD, studies reporting hippocampal shape deformation are rare. ${ }^{8,32-34}$

Recently, shape contractions representing regional atrophy of the hippocampus in patients with MDD have been reported in local regions of the subiculum and tail of the hippocampus, ${ }^{32}$ both anterior CA1-CA3 subfields, and the subiculum (the basal head of hippocampus ${ }^{33}$ in patients with depression. Hippocampal shape contractions have been reported in the left midbody and the lateral side near the head in patients with unremitting $\mathrm{MDD},{ }^{8}$ and depressed patients with Apoe E4 showed more severe shape contractions in the anterior CA1 than did patients with depression without Apoe E4. ${ }^{34}$ However, the findings from these previous studies are inconsistent; these inconsistencies could be the result of variations in the clinical factors of the depressed patients, such as age, sex, and disease severity or in the various methods of hippocampal segmentation, including anatomic boundary criteria and shapeanalysis algorithms. Therefore, these clinical and methodologic characteristics need to be considered to reach a consensus about hippocampal shape changes in patients with MDD.

In our study, the shape contractions of the left hippocampus were wider and more severe than the shape contractions of the right hippocampus. The results of the shape analysis corresponded with the results of the hippocampus volumetry, showing more severe atrophy in the left hippocampus (Table and Fig 3). Regional shape contractions were found in the ambient gyrus, the basal hippocampal head, the posterior subiculum, and the anterior and posterior dorsal hippocampus (CA2 and CA3) of the left hemisphere; the right hippocampus showed a similar pattern of shape contraction but was less atrophic compared with the left hippocampus. A negative correlation was also found between the HDRS scores and shape deformation in the CA3, ambient gyrus, and posterior subiculum (Fig 4).

Stress is known to be one of the factors that damages the hippocampus, hypothalamus, and pituitary, and it strongly suppresses the adult neurogenesis of the hippocampus by increasing glucocorticoid release. The decreased neurogenesis in the dentate gyrus is thought to be an important pathophysiologic factor of depression. 3,35-39

Most interesting, our analysis showed that the ambient gyri were contracted in both hippocampi, and the left ambient gyrus was more severely contracted than the right ambient gyrus (Fig 3A). The ambient gyrus, located at the rostral parahippocampal gyrus, is the junction between the anterior mesial temporal lobe and the amygdala. The shape contractions of ambient gyri imply that there could be problems in the interconnection between the hippocampus and amygdala. As well as hippocampal volume reduction in MDD, abnormal volume changes have been reported in the amygdala in MDD, and a recent meta-analysis concluded that the amygdala volume of medicated patients with MDD is increased compared with healthy controls, whereas the amygdala volume in unmedicated patients with MDD was decreased. The reason postulated for this difference is that antidepressant medication in- 
creased levels of brain-derived neurotrophic factors, promoting neurogenesis in the amygdala. ${ }^{40}$

In this study, additionally, the shape contraction in the subiculum suggests an abnormal connection between CA1 and the entorhinal cortex (Fig 3). The subiculum is located at the inferomedial area of the hippocampus and has a connection between the CA1 and the entorhinal cortex. The decreased shape deformation of the posterior subiculum associated with increased HDRS scores may imply that the abnormal posterior hippocampus could be related to the severity of major depression (Fig 4). ${ }^{22}$

It is still not clear why specific subfields such as the anterobasal CA1, dorsal CA2, CA3, posterior subiculum, and ambient gyrus of the hippocampus of patients with MDD were contracted in the shape analysis. However, according to a large number of reviews of previous studies, ${ }^{1,4,20,35-38,41}$ the loss of hippocampal volume can be explained by a number of hypotheses: 1) the effect of neurotoxicity induced by the hypersecretion of glucocorticoids possibly contributing to the neuronal death; 2) the failing adult neurogenesis in the dentate gyrus, contributing to the weak neuronal projections from the dentate gyrus to the CA3 subfield, as an important causal factor for the deficits in these subfields; 3 ) the damage to dendrites; and 4) the loss of glial cells.

Although hippocampal shape analysis showed regional shape deformations in MDD effectively, a very high-resolution MR imaging enabling subregional shape analyses of the dentate gyrus, CA1, CA3, and the subiculum might be appropriate to understand the hippocampal abnormality in the specific subregions, particularly the abnormal neurogenesis of the dentate gyrus and its effect on hippocampal subregions. ${ }^{42}$

\section{Conclusions}

Our study identified atrophy and regional shape contractions in the hippocampus of patients with MDD and found that the atrophy and shape contraction were more significant in the left hippocampus. These results support the hypothesis that the hippocampus, particularly on the left side, is one of the brain structures playing an important role in the pathogenesis of major depressive disorders.

\section{References}

1. Sapolsky RM. The possibility of neurotoxicity in the hippocampus in major depression: a primer on neuron death. Biol Psychiatry 2000;48:755-65

2. Yanike M, Wirth S, Suzuki WA. Representation of well-learned information in the monkey hippocampus. Neuron 2004;42:477-87

3. Jacobs BL, Praag H, Gage FH. Adult brain neurogenesis and psychiatry: a novel theory of depression. Mol Psychiatry 2000;5:262-69

4. Lee AL, Ogle WO, Sapolsky RM. Stress and depression: possible links to neuron death in the hippocampus. Bipolar Disord 2002;4:117-28

5. Campbell S, Marriott M, Nahmias C, et al. Lower hippocampal volume in patients suffering from depression: a meta-analysis. Am J Psychiatry 2004;161:598-607

6. Videbech P, Ravnkilde B. Hippocampal volume and depression: a meta-analysis of MRI studies. Am J Psychiatry 2004;161:1957-66

7. Styner M, Gerig G, Lieberman J, et al. Statistical shape analysis of neuroanatomical structures based on medial models. Med Image Anal 2003;7:207-20

8. Zhao Z, Taylor WD, Styner M, et al. Hippocampus shape analysis and late-life depression. PLoS One 2008;3:e1837

9. Tae WS, Kim SS, Lee KU, et al. Validation of hippocampal volumes measured using a manual method and two automated methods (FreeSurfer and IBASPM) in chronic major depressive disorder. Neuroradiology 2008;50:569-81

10. Steinberg M. Interviewers Guide to the Structured Clinical Interview for DSM-IV
Dissociative Disorders (SCID-D). Washington, DC: American Psychiatric Press; 1994

11. Beck AT, Ward CH, Mendelson M, et al. An inventory for measuring depression. Arch Gen Psychiatry 1961;4:561-71

12. Hamilton M. A rating scale for depression. J Neurol Neurosurg Psychiatry 1960;23:56-62

13. Eritaia J, Wood SJ, Stuart GW, et al. An optimized method for estimating intracranial volume from magnetic resonance images. Magn Reson Med 2000;44:973-77

14. Yushkevich PA, Piven J, Hazlett HC, et al. User-guided 3D active contour segmentation of anatomical structures: significantly improved efficiency and reliability. Neuroimage 2006;31:1116-28

15. Cronbach LJ. Coefficient alpha and the internal structure of tests. Psychometrika 1951;16:297-334

16. Styner M, Lieberman JA, Pantazis D, et al. Boundary and medial shape analysis of the hippocampus in schizophrenia. Med Image Anal 2004;8:197-203

17. Jernigan TL, Gamst AC, Fennema-Notestine C, et al. More "mapping" in brain mapping: statistical comparison of effects. Hum Brain Mapp 2003;19:90-95

18. Jenkins R, Fox NC, Rossor AM, et al. Intracranial volume and Alzheimer's disease: evidence against the cerebral reserve hypothesis. Arch Neurol 2000;57:220-24

19. Fein G, Di Sclafani V, Taylor C, et al. Controlling for premorbid brain size in imaging studies: T1-derived cranium scaling factor vs. T2-derived intracranial vault volume. Psychiatry Res 2004;131:169-76

20. Kruggel F. MRI-based volumetry of head compartments: normative values of healthy adults. Neuroimage 2006;30:1-11

21. MacQueen GM, Campbell S, McEwen BS, et al. Course of illness, hippocampal function, and hippocampal volume in major depression. Proc Natl Acad Sci U S A 2003;100:1387-92

22. Maller JJ, Daskalakis ZJ, Fitzgerald PB. Hippocampal volumetrics in depression: the importance of the posterior tail. Hippocampus 2007;17:1023-27

23. O'Brien JT, Lloyd A, McKeith I, et al. A longitudinal study of hippocampal volume, cortisol levels, and cognition in older depressed subjects. Am J Psychiatry 2004;161:2081-90

24. Crow TJ. Schizophrenia as failure of hemispheric dominance for language. Trends Neurosci 1997;20:339-43

25. Mackay CE, Barrick TR, Roberts N, et al. Application of a new image analysis technique to study brain asymmetry in schizophrenia. Psychiatry Res 2003;124:25-35

26. Allen JS, Bruss J, Mehta S, et al. Effects of spatial transformation on regional brain volume estimates. Neuroimage 2008;42:535-47

27. Shattuck DW, Mirza M, Adisetiyo V, et al. Construction of a 3D probabilistic atlas of human cortical structures. Neuroimage 2008;39:1064-80

28. Bhatia S, Bookheimer SY, Gaillard WD, et al. Measurement of whole tempora lobe and hippocampus for MR volumetry: normative data. Neurology 1993;43:2006-10

29. Bigler ED, Blatter DD, Anderson CV, et al. Hippocampal volume in normal aging and traumatic brain injury. AJNR Am J Neuroradiol 1997;18:11-23

30. Anstey KJ, Maller JJ, Meslin C, et al. Hippocampal and amygdalar volumes in relation to handedness in adults aged 60-64. Neuroreport 2004;15:2825-29

31. Raz N, Gunning-Dixon F, Head D, et al. Aging, sexual dimorphism, and hemispheric asymmetry of the cerebral cortex: replicability of regional differences in volume. Neurobiol Aging 2004;25:377-96

32. Posener JA, Wang L, Price JL, et al. High-dimensional mapping of the hippocampus in depression. Am J Psychiatry 2003;160:83-89

33. Ballmaier M, Narr KL, Toga AW, et al. Hippocampal morphology and distinguishing late-onset from early-onset elderly depression. Am J Psychiatry 2008;165:229-37

34. Qiu A, Taylor WD, Zhao Z, et al. APOE-related hippocampal shape alteration in geriatric depression. Neuroimage 2009;44:620-26

35. Bremner JD. Does stress damage the brain? Biol Psychiatry 1999;45:797-805

36. Jacobs BL. Adult brain neurogenesis and depression. Brain Behav Immun 2002;16:602-09

37. Swaab DF, Bao AM, Lucassen PJ. The stress system in the human brain in depression and neurodegeneration. Ageing Res Rev 2005;4:141-94

38. Warner-Schmidt JL, Duman RS. Hippocampal neurogenesis: opposing effects of stress and antidepressant treatment. Hippocampus 2006;16:239-49

39. Bao AM, Meynen G, Swaab DF. The stress system in depression and neurodegeneration: focus on the human hypothalamus. Brain Res Rev 2008;57:531-53

40. Hamilton JP, Siemer M, Gotlib IH. Amygdala volume in major depressive disorder: a meta-analysis of magnetic resonance imaging studies. Mol Psychiatry 2008;13:993-1000

41. Kempermann G, Krebs J, Fabel K. The contribution of failing adult hippocampal neurogenesis to psychiatric disorders. Curr Opin Psychiatry 2008;21:290-95

42. Yushkevich PA, Avants BB, Pluta J, et al. A high-resolution computational atlas of the human hippocampus from postmortem magnetic resonance imaging at 9.4 T. Neuroimage 2009;44:385-98 